\title{
Lessons Learned from COVIDSafe: Understanding Conditions for Successful Implementation of Track and Trace Technologies
}

\section{Nathaniel Carpenter and Anna Dabrowski}

Digital track and trace works not just because of cutting edge technology, but because of public trust - a key condition for its utility. We have seen that trust is fundamental to other government COVID-19 tracking programs. This should be cultivated in Australia with engagement of experts and academics to provide a clear public message.

\subsection{Introduction}

As a consequence of the current COVID-19 pandemic, many national governments have retroactively deployed digital track and trace systems to support ongoing community health monitoring. In some contexts, contact tracing technologies have met with great success in monitoring the spread of the novel coronavirus, while in other spaces, there has been failure in regards to both implementation and uptake. This chapter focuses on the implementation of COVIDSafe, a track and trace technology designed for the Australian context. Building upon lessons learned from the successful implementation of contact tracing mechanisms in Asia, we argue that the success of contact tracing mechanisms depends not only on the quality of tracing technology, but on the cultural conditions of government and community that surround their enactment.

\subsection{Do track and Trace Mechanisms Work?}

Contact tracing technologies can provide communities with a method to contain the spread of communicable diseases by quickly identifying and notifying people who have come into contact with infected individuals [1]. Exposed individuals could then be informed, tested, and isolated, which would break the chain of further transmission. However, breaking the chain only works if individuals both use and trust the system while also adhering to self-isolating advice. It is also important to remember that track and trace mechanisms do not actually render individuals safe from the novel coronavirus. Although contact tracing should (in theory) support community awareness and protection, contact tracing alone does not offer inoculation like a vaccination might $[2]$. 
Effective uses of contact tracing tend to be found in East Asia, such as China, South Korea, Taiwan, Singapore and to a lesser extent, Japan [3, 4]. Yet the reason for the success of track and trace technologies in Asian nations may be cultural, as opposed to technological. In addition to developing technologies to monitor the population, many Asian nations have previous experience with epidemics such as MERS and SARS. Trust in government underpins civic duty and collectivist cultures, which often embrace compliance with government infection control mechanisms. Contact tracing applications in countries like South Korea are also mandated and invasive, yet extremely popular, and discussed widely by community members. In contrast, most western democracies have had little experience with modern infection control tracing techniques [5-7] which include adopting technology, implementing infrastructure, and streamlined data sharing agreements; let alone public awareness campaigns on contact tracing.

\subsection{The Failures of COVIDSafe: Technology or User?}

Despite the name, COVIDSafe has not succeeded in protecting the Australia public from COVID-19. However, the failures of this contact tracing mechanism can be attributed to several key factors that extend beyond the technological [8-10]. A lack of trust in government, coupled with a rushed response to developing the tool, has certainly led to a lack of public investment in the implementation of COVIDSafe. Mixed messages around the efficacy and safety of the tool has led to a belief of limited benefit, and individualistic culture has undermined Australia's efforts to implement their own track and trace mechanism successfully.

Inexperience in secure enactment of track and trace technology has also marred public perception of the efficacy of COVIDSafe. Australia lacks the experience of its Asian counterparts: even in May 2020, well after the novel coronavirus had developed into a pandemic, infrastructure was still not in place for the Australian government to act on notifications of infected individuals and their contacts. One major concern relates to data security [9]. In the case of COVIDSafe, if a user tests positive for COVID-19 and consents to their data being uploaded, the information is then held by the federal government on an Amazon Web Services server in Australia. Data from the app is stored on a user's device and transmitted in an encrypted form to the cloud-based server. Cloud based storage poses risks at the best of times [11]; unfortunately, Australia also has a long history of compromised data usage. Concerns around privacy render many members of the Australian public reticent to utilise basic health storage technologies, such as MyHealthRecord [12], let alone invasive track and trace technologies. Like financial data, spatial data is sensitive, identifiable information able to compromise an individual's identity and safety.

Although COVIDSafe does not access positioning information, it does ask for permission to collect information about accessing the phone network, which can be used as a form of location tracking. COVIDSafe uses Bluetooth to record anyone who is within range of the signal [10]. This concept does allow people to remain spatially anonymous; however, since most individuals' routines are fairly stable and most people have a physical contact group which tends to remain the same, it would not be difficult to infer locations and associations. Importantly, based on previous data security breaches [13], the Australian public is aware of vulnerability in data collection systems, and their ability to be exploited. Privacy implications remain murky. Evidence from the Australian public highlights issues with platforms and device error [9], often resulting in users uninstalling COVIDSafe. Another major issue is that individuals must upload their information through a rather complicated process; perhaps this is why individuals who test positive to COVID-19 fail to report. 


\subsection{Enhancing Implementation Through Education}

There are many ways to enhance the utility of track and trace mechanisms, and success should not only be seen as culturally determined. For while South Korea and China have successfully employed contact tracing mechanisms [14], there have also been low levels of success in track and trace technologies in certain neighbouring contexts, such as Singapore. However, without public support of digitally enabled track-and-trace technologies, the purported digital inoculation will fall short of intended goals [15], be it low number of users such as seen in countries such as Singapore [16], or in an overall reduction in transmission rates [17].

Although it is crucial to improve user uptake, increasing awareness through broader education is also important, particularly to overcome a lack of community understanding as to the limits of contact tracing technology (see [18]). Awareness campaigns that raise knowledge and understanding are important tools for promoting change in social behaviours and norms; however, an important consideration is understanding the cultural context [19]. Raising awareness of track and trace processes is crucial, but assurances of data security and transparency are also necessary steps to building trust and overcoming public cynicism as to the utility of contact tracing tools. However, if governments fail to take into consideration that a country like Australia promotes a culture of self over community, it is unlikely that track and trace systems can ever succeed.

\subsection{Lessons from Australia: Enhancing Contact Tracing}

There are many reasons why track and trace mechanisms fail. In this chapter, we have reflected on the lessons learned from Australia, as evidenced in the example of Australia's COVIDSafe tool. While community hesitation and a lack of willingness to download the application can be attributed to an absence of trust towards the government, the manner of implementation is also of concern. A lack of public education and trust has failed to generate a meaningful awareness of what COVIDSafe can (and cannot do) in terms of providing protection for the Australian population. As a consequence, in order for COVIDSafe (or future track and trace technologies) to be successful, it is crucial for government efforts to focus on enhancing the capabilities of tracing technologies and providing an awareness which addresses community concerns. Increasing knowledge is the first step to behavioural change [15], however, being responsive and building trust will also help the Australian government to navigate the risks posed by this pandemic, and the next.

\section{References}

[1] Joel Hellewell, Sam Abbott, Amy Gimma, Nikos I Bosse, Christopher I Jarvis, Timothy W Russell, James D Munday, Adam J Kucharski, W John Edmunds, Fiona Sun, Stefan Flasche, Billy J Quilty, Nicholas Davies, Yang Liu, Samuel Clifford, Petra Klepac, Mark Jit, Charlie Diamond, Hamish Gibbs, Kevin van Zandvoort, Sebastian Funk, and Rosalind M Eggo. Feasibility of controlling COVID-19 outbreaks by isolation of cases and contacts. The Lancet Global Health, 8(4):e488-e496, 2020. ISSN 2214-109X. doi: https://doi.org/10. 1016/S2214-109X(20)30074-7.

[2] Danielle Currie, Cindy Peng, David Lyle, Brydie Jameson, and Michael Frommer. Stemming the flow: How much can the Australian smartphone app help to control COVID-19? Public Health Research ES Practice, 30(2), 2020. doi: 10.17061/phrp3022009.

[3] Kin On Kwok, Arthur Tang, Vivian W. I. Wei, Woo Hyun Park, Eng Kiong Yeoh, and Steven Riley. Epidemic Models of Contact Tracing: Systematic Review of Transmission Studies of Severe Acute Respiratory Syndrome and Middle East Respiratory Syndrome. Computational and Structural Biotechnology Journal, 17:186-194, Jan 2019. ISSN 2001-0370. doi: 10.1016/j.csbj.2019.01.003. 
[4] Juhwan Oh, Jong-Koo Lee, Dan Schwarz, Hannah L. Ratcliffe, Jeffrey F. Markuns, and Lisa R. Hirschhorn. National Response to COVID-19 in the Republic of Korea and Lessons Learned for Other Countries. Health Systems \& Reform, 6(1):e1753464, 2020. doi: 10.1080/23288604.2020.1753464.

[5] Sreeram Chaulia. Why East beats West in the war against coronavirus. 2020.

[6] Maria Savona. The Saga of the Covid-19 Contact Tracing Apps: Lessons for Data Governance. 2020.

[7] Max Fisher and Choe Sang-Hun. How South Korea Flattened the Curve. The New York Times, 2020.

[8] Kobi Leins, Chris Culnane, and Benjamin I. P. Rubinstein. Tracking, tracing, trust: contemplating mitigating the impact of COVID-19 through technological interventions. The Medical Journal of Australia, 213(1):6-8, 2020. doi: $10.5694 / \mathrm{mja} 2.50669$.

[9] Rae Thomas, Zoe Michaleff, Hannah Greenwood, Eman Abukmail, and Paul Glasziou. More than privacy: Australians' concerns and misconceptions about the COVIDSafe App: a short report. medRxiv, 2020. doi: 10.1101/2020.06.09.20126110.

[10] David Watts. COVIDSafe, Australia's Digital Contact Tracing App: The Legal Issues (May 2, 2020). Australia's Digital Contact Tracing App, 2020. doi: http://dx.doi.org/10.2139/ssrn.3591622.

[11] Justin Chan, Dean Foster, Shyam Gollakota, Eric Horvitz, Joseph Jaeger, Sham Kakade, Tadayoshi Kohno, John Langford, Jonathan Larson, Puneet Sharma, Sudheesh Singanamalla, Jacob Sunshine, and Stefano Tessaro. PACT: Privacy Sensitive Protocols and Mechanisms for Mobile Contact Tracing, 2020.

[12] Timothy Kariotis, Megan Prictor, Shanton Chang, and Kathleen Gray. Evaluating the Contextual Integrity of Australia's My Health Record. Studies in health technology and informatics, 265:213-218, Aug 2019. ISSN 0926-9630. doi: 10.3233/shti190166.

[13] Randike Gajanayake, Bill Lane, Tony Iannella, and Tony Sahama. Legal issues related to Accountable-eHealth systems in Australia. 2012. doi: https://doi.org/10.4225/75/5796f5b740a96.

[14] Vincent Chi-Chung Cheng, Josepha Wai-Ming Tai, Ng Lai-Ming, Jasper Chan, Sally Wong, Iris Li, Hon-Ping Chung, Wai-Kei Lo, Kwok-Yung Yuen, and Pak-Leung Ho. Extensive contact tracing and screening to control the spread of vancomycin-resistant Enterococcus faecium ST414 in Hong Kong. Chinese Medical Journal, 125(19):3450-3457, 2012. doi: 10.3760/cma.j.issn.0366-6999.2012.19.017.

[15] John Hawkins and Ben Freyens. Contact tracing apps: a behavioural economist's guide to improving uptake. The Conversation, 2020:1-7, April 2020.

[16] Lucy Simko, Ryan Calo, Franziska Roesner, and Tadayoshi Kohno. Covid-19 contact tracing and privacy: Studying opinion and preferences. arXiv preprint arXiv:2005.06056, 2020.

[17] Andrea Nuzzo, Can Ozan Tan, Ramesh Raskar, Daniel C. DeSimone, Suraj Kapa, and Rajiv Gupta. Universal Shelter-in-Place Versus Advanced Automated Contact Tracing and Targeted Isolation: A Case for 21st-Century Technologies for SARS-CoV-2 and Future Pandemics. Mayo Clinic Proceedings, 95(9):1898 - 1905, 2020. ISSN 0025-6196. doi: https://doi.org/10.1016/j.mayocp.2020.06.027.

[18] Leila Pfaeffli Dale, Lauren White, Marc Mitchell, and Guy Faulkner. Smartphone app uses loyalty point incentives and push notifications to encourage influenza vaccine uptake. Vaccine, 37(32):4594-4600, 2019. ISSN 0264-410X. doi: https://doi.org/10.1016/j.vaccine.2018.04.018. URL http://www.sciencedirect.com/ science/article/pii/S0264410X18304870.

[19] Linda Brennan, Wayne Binney, Lukas Parker, Torgeir Aleti (né Watne), and Dang Nguyen. Social Marketing and Behaviour Change: Models, Theory and Applications, 11, 2014. ISBN 1782548157, 9781782548157. doi: $10.4337 / 9781782548157$. 